EFDA-JET-CP(09)03/02

C. Lescure, G. Fusiek, S. Hotchin, E. Ivings, M.F. Johnson, P. Niewczas, V. Riccardo, M. Walsh, A. West and JET EFDA contributors

\title{
Measurement of Disruption Forces in JET using Fiber-Optic Sensors
}


"This document is intended for publication in the open literature. It is made available on the understanding that it may not be further circulated and extracts or references may not be published prior to publication of the original when applicable, or without the consent of the Publications Officer, EFDA, Culham Science Centre, Abingdon, Oxon, OX14 3DB, UK."

"Enquiries about Copyright and reproduction should be addressed to the Publications Officer, EFDA, Culham Science Centre, Abingdon, Oxon, OX14 3DB, UK."

The contents of this preprint and all other JET EFDA Preprints and Conference Papers are available to view online free at www.iop.org/Jet. This site has full search facilities and e-mail alert options. The diagrams contained within the PDFs on this site are hyperlinked from the year 1996 onwards. 


\section{Measurement of Disruption Forces in JET using Fiber-Optic Sensors}

C. Lescure $^{1}$, G. Fusiek ${ }^{2}$, S. Hotchin ${ }^{1}$, E. Ivings ${ }^{1}$, M.F. Johnson ${ }^{1}$, P. Niewczas ${ }^{2}$, V. Riccardo ${ }^{1}, \mathrm{M}$. Walsh ${ }^{1}, \mathrm{~A}$. West ${ }^{1}$ and JET EFDA contributors*

JET-EFDA, Culham Science Centre, OX14 3DB, Abingdon, UK

${ }^{I}$ EURATOM-UKAEA Fusion Association, Culham Science Centre, OX14 3DB, Abingdon, OXON, UK

${ }^{2}$ University of Strathclyde 204 George Street, Glasgow, G1 1XW, UK

* See annex of $F$. Romanelli et al, "Overview of JET Results",

(Proc. $22^{\text {nd }}$ IAEA Fusion Energy Conference, Geneva, Switzerland (2008)).

Preprint of Paper to be submitted for publication in Proceedings of the

36th International Conference on Plasma Science and

23rd Symposium on Fusion Engineering Conference, ICOPS-SOFE 2009

San Diego, USA

(31st May 2009 - 5th June 2009) 



\begin{abstract}
Monitoring the forces induced on the vacuum vessel when a disruption occurs is essential for the protection of the machine. Since divertor coils have been installed in JET, during disruptions a significant difference between the forces measured on the top and the bottom of the machine has been observed. In order to investigate these further, optical fiber transducers Fiber Bragg Gratings (FBGs) were installed in addition to the currently used strain gauges. During Vertical Displacement Event (VDE) tests, these new transducers were used to measure different level of disruptions ranging from $80 \mathrm{~T}$ up to $230 \mathrm{~T}$ vertical forces. The results were then compared with the strain gauges. The FBG measurement was carried out using a broadband light source illuminating an array of 4 FBGs through a combination of directional couplers, connectors and fiber-optic cable. The forces measured both by FBG and by the strain gauges are within the same range, except for the initial swing where large random differences are observed. The tests have demonstrated that the FBG-based force measurement system has adequate resolution $( \pm 1.5 \mu$ strain corresponding to a force of $4 \mathrm{kN}$ due to the geometry of the leg) and measurement range $(5000 \mu$ strain or $13000 \mathrm{kN})$ for this application.
\end{abstract}

\title{
1. FORCES MEASUREMENT IN JET
}

Vessel forces measurement in JET is part of the Machine Diagnostic System (MDS), this system also includes displacement measurements at different points of the machine [1]. Measurements of the forces are made via Strain Gauges (SG) placed on each vessel restraints support (legs) of the machine. The JET machine is divided into 8 octants, there are four supports to each octant, $(2$ on the top and 2 on the bottom) oriented at about 45 degrees from the vertical (Fig.1).

The legs are attached to the main vertical ports and connected to the iron limbs via brakes systems. During plasma operation the brakes are clamped, they are released during vessel temperature transients, as well as for shutdown and standby. For redundancy purposes each leg is served by 2 strain gauges measuring the same force. Data are continually monitored and constitutes an additional check to make sure that the brakes are off when the temperature cycle of the machine takes place. During disruptions the force measurements are sampled at $2.5 \mathrm{kHz}$ and stored in the acquisition system for the vertical force calculation and further analysis. Signals from strain gauges are also sampled at a slow rate $(25 \mathrm{~Hz})$ for the duration of the plasma discharge recording static forces due the current flowing in the coils.

For safe JET operation, before a newly developed plasma scenario is allowed to be used at high plasma current ( $>3 \mathrm{MA}$ ), the disruption force has to be assessed during commissioning (VDE test), as disruption forces could be harmful for the machine. If a disruption occurs the forces calculated are compared with the expected value, if a difference is too large then careful consideration would have to take place in order to carry on the operation.

\section{TRADITIONAL STRAIN GAUGES}

Strain gauges installed on the legs in the 1990s use foiled type 4 wire transducers. All strain gauges 
are routed to termination cubicles and then to signal conditioning cubicles after which they are passed to a data acquisition system. A conditioning module energizes a transducer with a stable ac $5 \mathrm{kHz}$ waveform and converts the output of the transducer to a dc voltage proportional to the strain. During disruption events the data is stored over a 2 second time window. The triggering is done via the JET CTS (Central Timing System)

A significant difference between the signals from transducers situated on the top of the vessel with those situated on the bottom is seen in disruption events. This became noticeable after the installation of the divertors in 1993. In order to understand fully this anomaly, further analysis has been done using also the displacement transducers of the intermediate vertical port. This analysis revealed a poor correlation between the right and the left top strain gauges, and also between the absolute upper vertical force and the absolute upper displacement transducers. The latter is shown in fig. 2 for the top and fig. 3 for the bottom. Hookes law determines that the relationship should be linear, this is observed for the bottom, but not for the top. The curve is consistent with additional frictional force acting on the legs such as semi-locked brakes with a lesser degree of freedom for larger forces; however there could be other causes for this effect such as poor instrumentation.

In order to investigate this further, a new type of sensors has been installed in the same locations to allow a direct signal comparison. Because of the electromagnetic nature of the environment an optical type of transducer was chosen namely a Fiber Bragg Grating (FBG). The sensors were placed on the vessel restraints using epoxy in a similar way to the strain gauges and measuring the same direction of the force. As part of the trial, 4 fibers have been fixed on the vessel support of octant 6 of the machine.

\section{FBG INSTALLATION}

\subsection{INSTALLATION OVERVIEW}

The new sensor test was conducted in one octant (4 FBGs). Because of its proximity to an open floor penetration, octant 6 was chosen. Before installing the sensors on the restraints, pigtail fibers had to be spliced to the bare end of the FBG.

A small area of the leg restraint was resurfaced to ensure a good contact between the fiber and the metal. Fibers were then bonded to the leg and a metal enclosure was fitted around the leg in order to avoid any possible damage to the sensor during weekend work. Both fibers on the top were connected in series to a connection box attached on the limbs. A single light source from the interrogation unit was connected to 2 fiber cables using an optical coupler (Fig.4).

The following picture (Fig.5) illustrates how the fibers are mounted to the vessel restraints. The instrumentation is linked with the sensors by 2 individual 80 -meter long optical fibers. The FBGs on the top and the bottom are connected in series. Each sensor has its own narrow band reflection wavelength enabling the discrimination between each measurement. An optical coupler was used to split light from the interrogation system into the top and bottom sensors. 


\subsection{MEASUREMENT SYSTEM OVERVIEW}

A Super-Fluorescent light Source (SFS), providing up to $30 \mathrm{~mW}$ of continuous power in the telecoms $\mathrm{C}$-Band was employed by the interrogator. The source was used to illuminate $4 \mathrm{FBG}$ sensors via an optical coupler and two separate 80-m fiber-optic cables (Fig.6). The cables contained multiple fibers, but only one fiber per line was used in this trial. Two fiber-optic connection boxes were also mounted for convenient connecting of the sensors, one at the bottom and one near the top of the JET machine (not shown in Figure 6).

The wavelength encoded signals returning from the FBGs were mixed in the coupler and analyzed using a tunable Fabry-Perot filter. The filter was driven using a saw-tooth signal to allow repeatable scanning over the required bands. To increase the scanning speed, detailed scanning was performed around the FBG peaks and coarse scanning outside the spectral regions of interest. The details of such selective scanning are given elsewhere [2]. The optical power at the scanning filter output was converted to an electrical signal using a photo-receiver module. The electrical signal was then analyzed using a National Instruments PXI system, and the peak wavelengths returned by the FBGs could be observed on the screen and recorded following the reception of a digital trigger signal from Control and Data Acquisition System (CODAS). A double-line peak detection technique was used to recover FBG peaks [2]. Using the selective scanning technique, the scanning frequency of $1.2 \mathrm{kHz}$ for all four FBGs was achieved. At this scanning frequency and the achieved power budget, the resolution was below $2 \mu$ Strain, for the bottom FBGs and the top-right FBG. The top-left FBG had a faulty pigtail splice, which resulted in poorer resolution, which amounted to approximately $6 \mu$ Strain.

The interrogation system was placed outside the biological shield, some $80 \mathrm{~m}$ away from the sensors.

\section{MEASUREMENT USING FBG}

Several levels of total vertical forces have been achieved ranging from 80T to 230T. Since the force is spread over 32 legs, each sensor only sees a fraction of this level.

\subsection{STRAIN MEASUREMENT:}

To eliminate any inaccuracy due to the potential interrogation system drift, a reference FBG having known peak wavelength, $\lambda_{\text {refFBG }}$, at $20^{\circ} \mathrm{C}$ was kept at constant temperature. The reference FBG peak position was recorded together with the peak wavelengths of the FBGs measuring the strain. An error between the measured and known peak wavelength of the reference FBG was calculated in the following way:

$$
\Delta \lambda_{\text {refFBG }}=\Delta \lambda_{\text {refFBG_recorded }}-\Delta \lambda_{\text {refFBG }}
$$

To eliminate noise from the resulting signal and to estimate the drift trend, a second order polynomial was fitted to the error data $\Delta \lambda_{\text {refFBG }}$. The recorded peak position of each FBG measuring strain was corrected by subtracting the drift trend, $\Delta \lambda_{\text {refFBG_drift }}$ :

$$
\lambda_{F B G}=\lambda_{\text {refFBG_recorded }}-\Delta \lambda_{\text {refFBG_drift }}
$$


Finally, the strain measured by an FBG was calculated using the following formula:

$$
S_{F B G}=\frac{\left(\lambda_{F B G}^{2}-\lambda_{F B G a v}\right.}{0.78 \cdot 10^{-6} \cdot \lambda_{F B G a v}}
$$

where $S_{F B G}=$ strain measured by an FBG, $\lambda_{F B G}=$ an FBG peak wavelength after drift elimination, $\lambda_{F B G a v}=$ the average of the first 200 samples of $\lambda_{F B G}$.

\subsection{FORCES CALCULATIONS:}

At the location of the FBG, the force is proportional to the Young's modulus of the leg material and the measure of the strain $\Delta \mathrm{L} / \mathrm{L}$. The leg is made of stainless steel which has a Young's modulus of $210,000 \mathrm{Mpa}$. The diameter of the leg is $11 \mathrm{~cm}$ giving a surface of $11.3 \times 10^{-3} \mathrm{~m}^{2}$. As the displacement variation is given in micro-strain, the resulting force relation is as follows:

$$
F_{(k N)}=1.996^{*} \varepsilon_{(\mu \text { Strain })}
$$

For comparison purposes data from the Strain Gauges measured in $\mathrm{kN}$ were converted in $\mu$ Strain.

\section{COMPARISONS BETWEEN SG AND FBG}

Looking at the signal received from the first step it becomes apparent that there was a good correlation between the strain gauges data and the FBG measurements for the major part of the signal in terms of amplitude of the signal and frequency of the oscillations, which validates the result from the FBG.

It is clear from the above graphs (Fig.7 and 8) that differences between forces at the top of the vacuum vessel and the bottom are similar to those recorded by the strain gauges. This also rules out any problems with the SGs.

Both fast data and continuous recording show very good agreement except for large spikes observed on all SG data in the initial phase of the disruption. These spikes are not present on the FBG measurement. The amplitude of the initial swing is used to calculate the overall vertical forces on the machine. Therefore the accuracy of this phase of the measurement is very important.

Overall, a vertical force is calculated using all 64 measurements on the machine, it is therefore possible that the error introduced by this initial large random spike is cancelled out. Nevertheless due to the large differences observed between the SG and the FBG (SG giving a swing amplitude of about 4 times higher than the FBG), it is conceivable that the result could be affected by this phenomenon.

The interpretation of these spikes can be attributed to one or both of the following:

- The SG cables are connected to the conditioning electronics via a plug situated near the vessel restraint. It is possible that during a disruption the connection mechanically moves making a variation to the overall resistance of the circuit which could lead to rather large spikes on the measurement. 
- The second possibility for producing spikes could be due to a rather large electro-magnetic fluctuation due to eddy currents and halo currents generated by the disruption interfering with the measurement.

As the light going through the optical fibers is not polarized and therefore immune to the electromagnetic interference, it would be then normal that those spikes are not seen by the FBG's. But this doesn't rule out a bad mechanical connection within the plug of the SG cable. However it is assumed that if that were to be the case, as the vibration of the machine continues for $500 \mathrm{~ms}$ after the event, we would still see spikes during all the vibration and not on the initial phase only.

Other differences between the FBGs and the SGs were seen during the continuous recording which has been performed during the daily dry run (commissioning pulse). The current flowing in the coils generates forces significant enough to be observed by the SG and the FBGs. On figure 10 some fluctuations can be seen on the SG when an imbalance current is applied into the P4 vertical field coils.

In the continuous recording mode, the FBGs were sampled at $500 \mathrm{~Hz}$, which offered a much better time resolution than the $\mathrm{SG}$ system $(25 \mathrm{~Hz})$. Due to the improved filtering on the SG system at $25 \mathrm{~Hz}$ sampling frequency, the noise level on the FBG signal was higher than that on the SG. In order to get to the same level of noise to compare the two signals, a 50-sample averaging on the FBG signal was implemented. As the oscillation from the $\mathrm{SG}$ is about $1 \mathrm{~Hz}$ this should still be observed on the FBG signal. However, those fluctuations were clearly not detected by the FBGs. This phenomenon cannot be explained due to a mechanical interference on the connection plug as we would then expect to see much larger random perturbations. This imbalance current is very low and produces very small electromagnetic changes in comparison to the one produced during a disruption

Further investigations, using the signals from the displacement transducers measuring the movement of the leg relative to the limbs in the same direction as the forces, demonstrates that those fluctuations are also not visible on the displacement signals (fig. 11).

Those displacements transducers are connected through the same connection as the strain gauges. This reinforces the suggestion that the SGs measurements are subject to electromagnetic perturbation.

\section{CONCLUSION}

The main objective of this work was to further understand the differences between the force recorded at the top and the bottom of the vessel and the bottom and also to check suitability of an FBG system in JET. The outcome of this work can be summarized as follows:

- By using a completely different type of transducer than the originally installed foil strain gauges this test revealed that the force measurements at the top and bottom of the machine are real as comparable signals are given by the FBG.

- This test also demonstrates that the force measurement in a machine like JET can be greatly improved by using the optical fiber sensor instead of the strain gauges.

- The speed of the response is comparable but the measurement accuracy appears to be much 
better as it is not subject to the electromagnetic interferences during the large variations of the magnetic field occurring during a disruption.

- The spikes observed on the initial swing of the disruption could affect the overall calculation of the vertical force. It would be therefore beneficial to install this type of measurement in JET on all octants. This would constitute several technical challenges especially with respect of the development of a suitable FBG interrogation system

\section{ACKNOWLEDGMENT}

This work was funded jointly by the UK Engineering and Physical Sciences Research Council and by the European Communities under the contract of Association between EURATOM and UKAEA. It was carried out within the framework of the European Fusion Development Agreement. The views and opinions expressed herein do not necessarily reflect those of the European Commission.

\section{REFERENCES}

[1]. V. Marchese, T. Businari, M. Buzio, E. de Marchi, N. Dolgetta, J. Howie, J. Last, T. Raimondi, L. Scibile, J. van Veen, "Enhancement of JET machine instrumentation and coil protection systems", Fusion Technology 1996. Proceedings of the 19th Symposium on Fusion Technology, Lisbon, Portugal, 16-20 September 1996. Vol.1. Amsterdam, North-Holland, 1997. p.747. Book shelved at FC SOFT 1996 Vol.1

[2]. G. Fusiek, P. Niewczas, J. R. McDonald, "Extended Step-out Length Fiber Bragg Grating Interrogation System for Condition Monitoring of Electrical Submersible Pumps", Optical Engineering, Vol. 44, No. 3, pp 034404-1-10, March 2005.

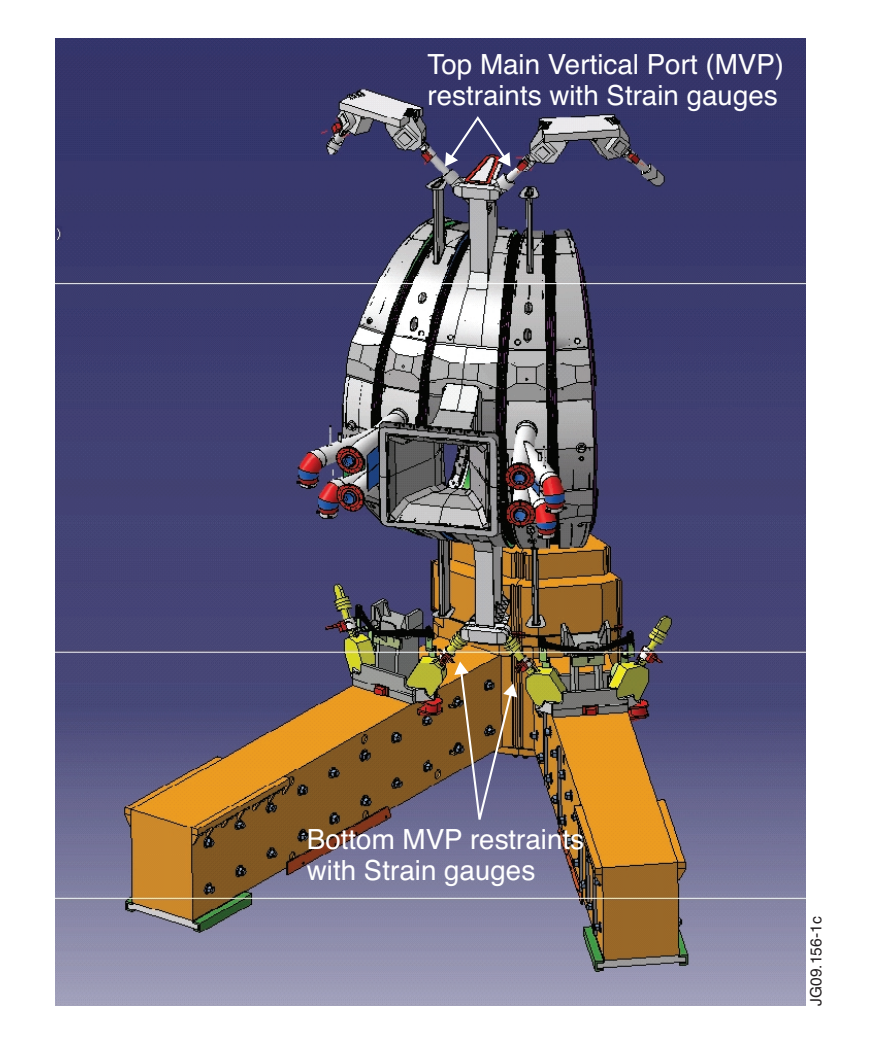

Figure 1: Restraints connected to the Main Vertical Port (MVP) of the JET vacuum vessel. 


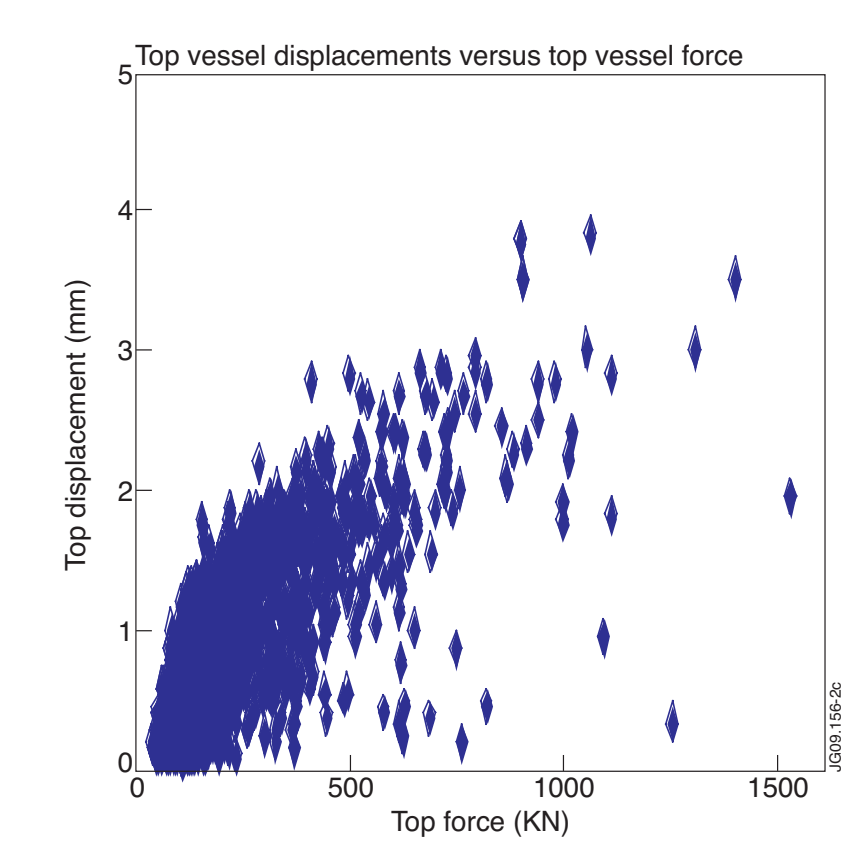

Figure 2

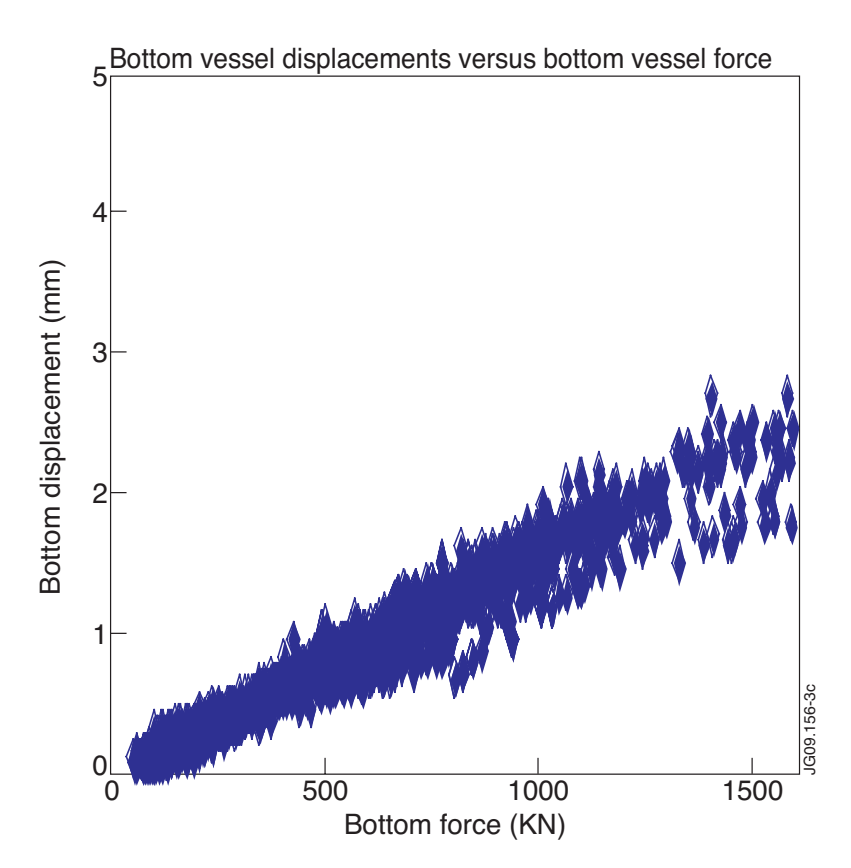

Figure 3

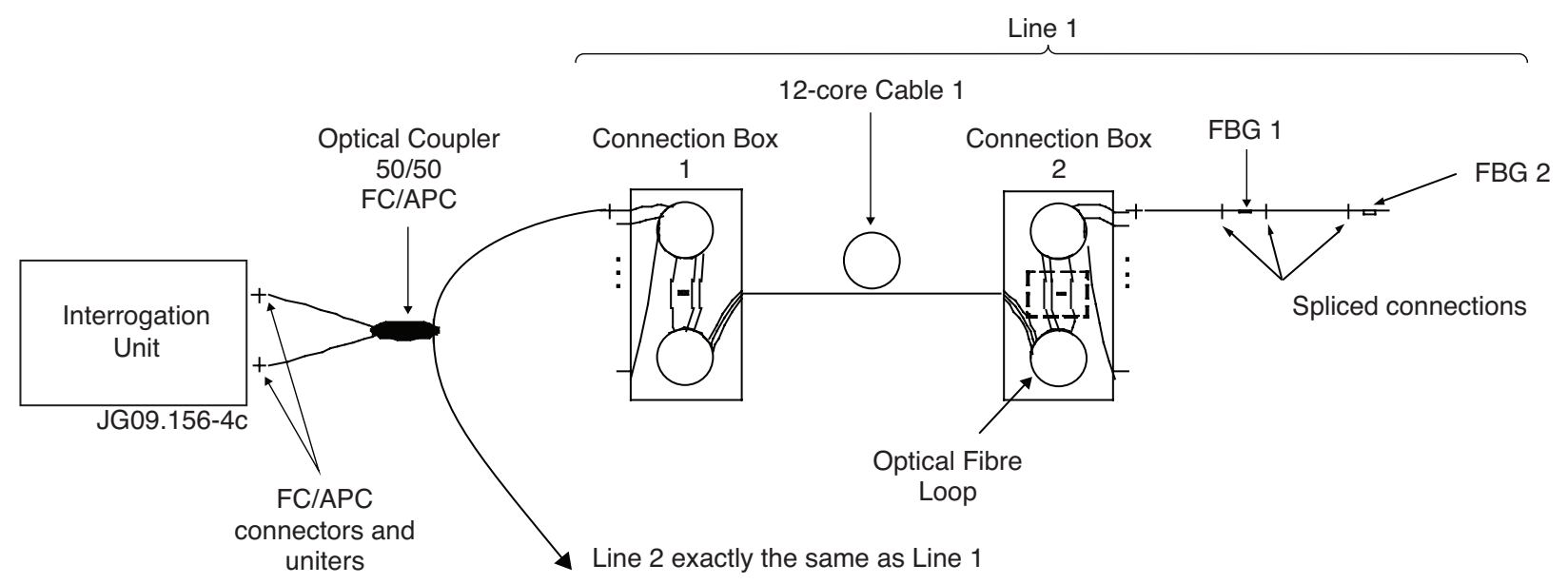

Figure 4: FBG arrangement.

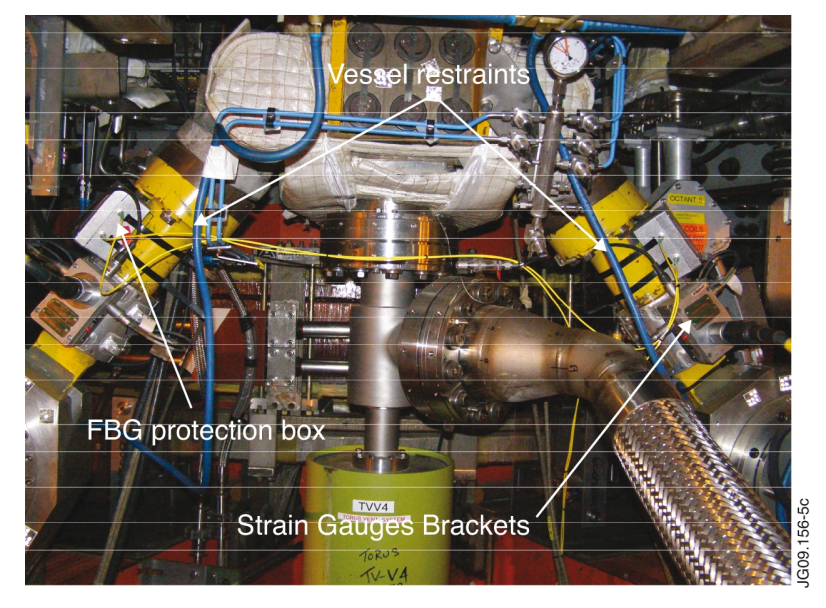

Figure 5: FBGs mounted on the top vessel restraints. 


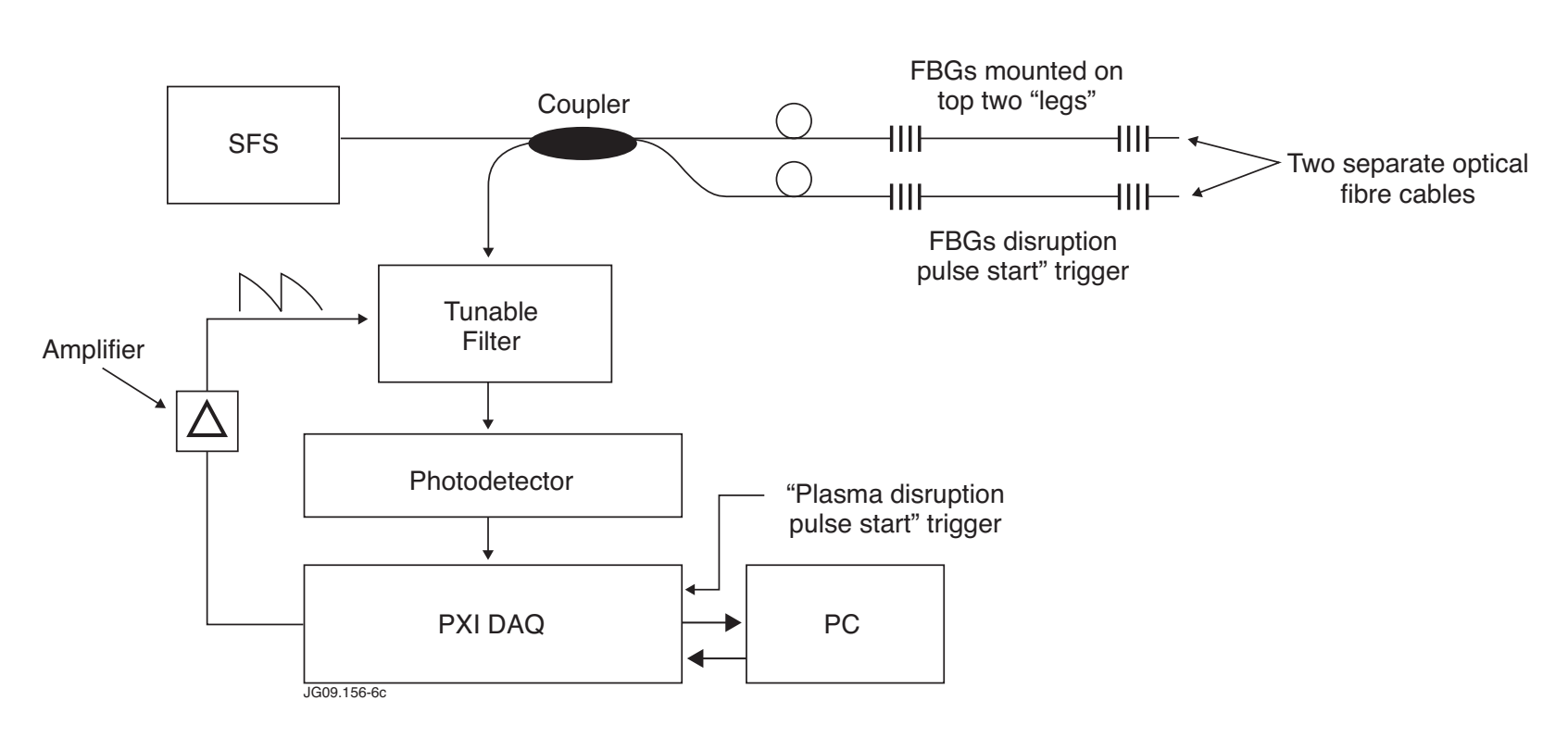

Figure 6: Schematic diagram of the FBG measurement system deployed on JET.

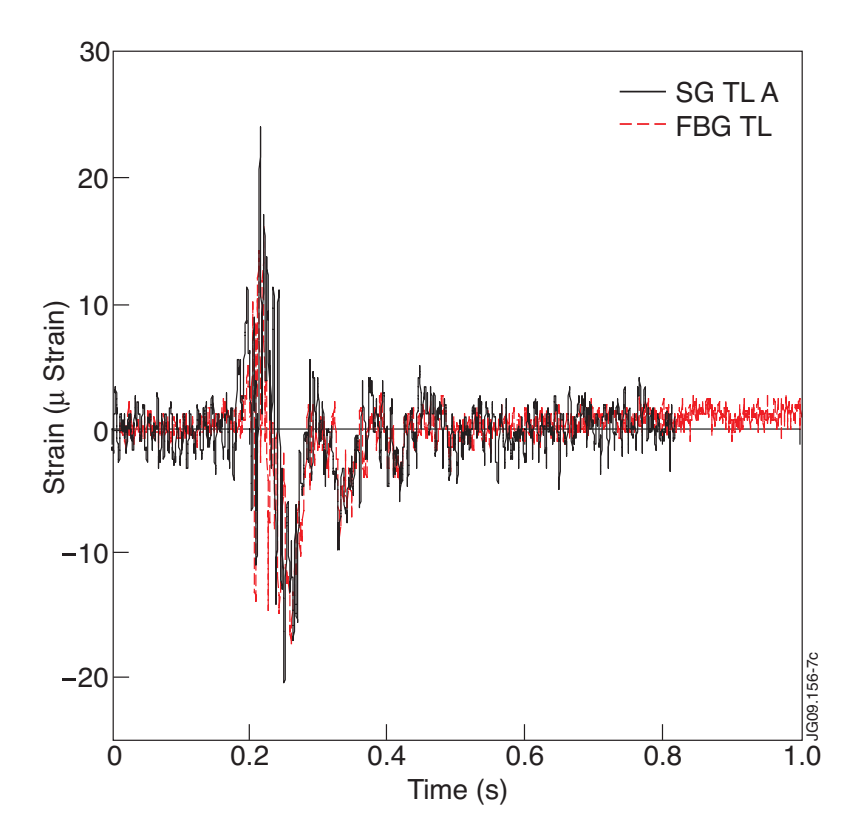

Figure 7: Top FBG versus SG, $230 T$ disruption.

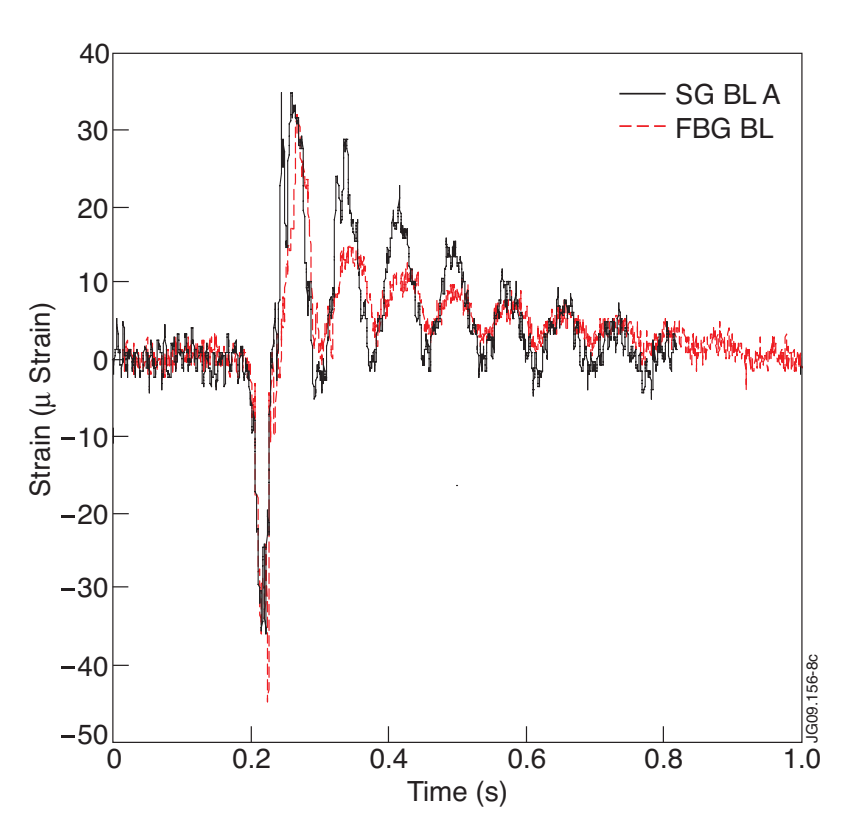

Figure 8: Bottom FBG versus SG, $230 T$ disruption. 


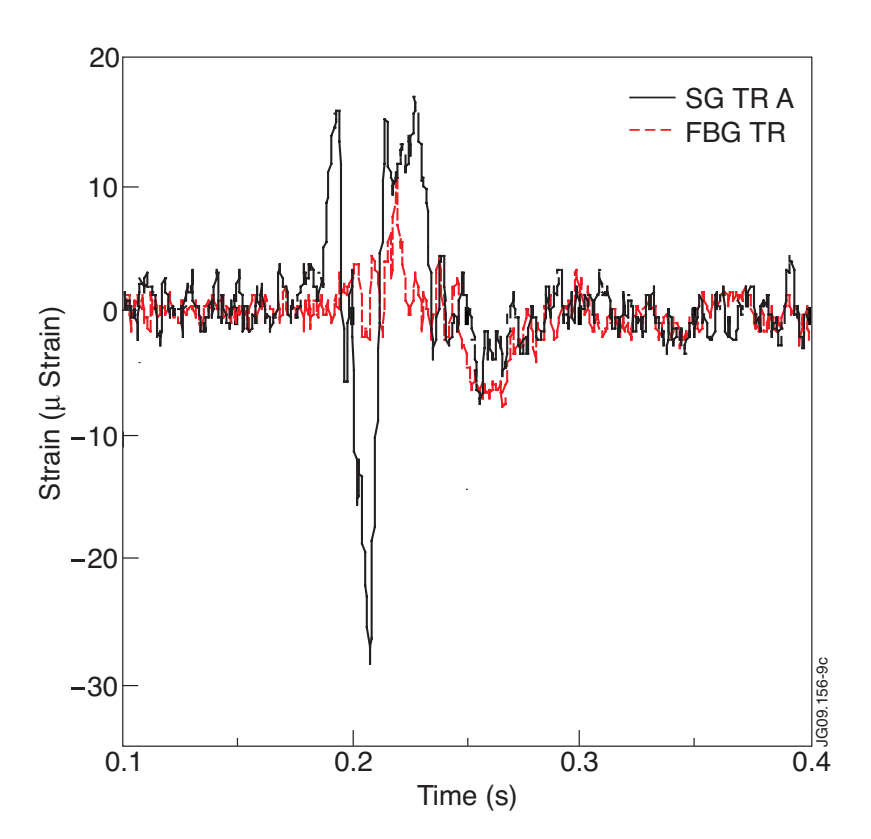

Figure 9: SG Spikes on initial disruption phase.
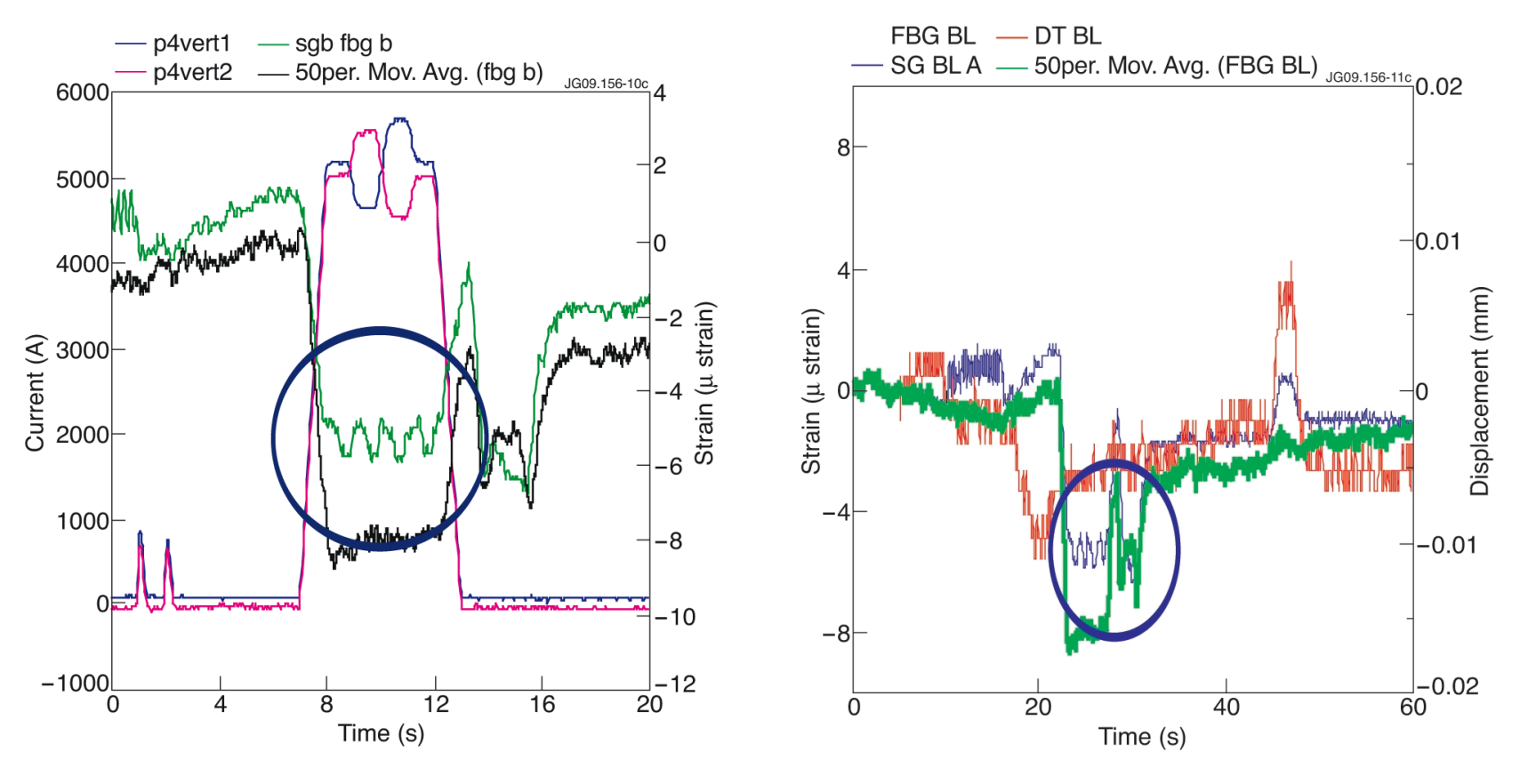

Figure 10: Forces measurements with vertical field

Figure 11: Forces measurements with legs displacement. imbalance. 